J. Nonlinear Var. Anal. 5 (2021), No. 1, pp. 103-118

Available online at http://jnva.biemdas.com

https://doi.org/10.23952/jnva.5.2021.1.07

\title{
FABER POLYNOMIAL COEFFICIENT ESTIMATES OF BI-CLOSE-TO-CONVEX FUNCTIONS CONNECTED WITH THE BOREL DISTRIBUTION OF THE MITTAG-LEFFLER TYPE
}

\author{
H.M. SRIVASTAVA ${ }^{1,2,3, *}$, G. MURUGUSUNDARAMOORTHY ${ }^{4}$, S.M. EL-DEEB ${ }^{5,6}$ \\ ${ }^{1}$ Department of Mathematics and Statistics, University of Victoria, Victoria, British Columbia V8W 3R4, Canada \\ ${ }^{2}$ Department of Medical Research, China Medical University Hospital, \\ China Medical University, Taichung 40402, Taiwan \\ ${ }^{3}$ Department of Mathematics and Informatics, Azerbaijan University, Baku, Azerbaijan \\ ${ }^{4}$ Department of Mathematics, School of Advanced Sciences, \\ Vellore Institute Technology University, Tamil Nadu, India \\ ${ }^{5}$ Department of Mathematics, Faculty of Science, Damietta University, New Damietta 34517, Egypt \\ ${ }^{6}$ Department of Mathematics, College of Science and Arts in Badaya, \\ Qassim University, Buraidah 51452, Saudi Arabia
}

\begin{abstract}
By using the Borel distribution series of the Mittag-Leffler type, we introduce a new class of the bi-close-to-convex functions defined in the open unit disk. We then apply the Faber polynomial expansion method in order to investigate the estimates for the general Taylor-Maclaurin coefficients of the functions belonging to this new class of bi-close-to-convex functions in the open unit disk. We consider the Fekete-Szegö type inequalities for the bi-close-to-convex function class and also consider several corollaries and the consequences of the results presented in this paper.

Keywords. Analytic, univalent and bi-univalent functions; Starlike, convex, close-to-convex and biclose-to-convex functions; Mittag-Leffler type Borel distribution; Faber polynomial expansion method; Fekete-Szegö type inequalities.
\end{abstract}

\section{INTRODUCTION, DEFINITIONS AND PRELIMINARIES}

Let $\mathscr{A}$ denote the class of functions of the form:

$$
f(z)=z+\sum_{k=2}^{\infty} a_{k} z^{k},
$$

which are analytic in the open unit disk $\Delta$ given by

$$
\Delta:=\{z: z \in \mathbb{C} \text { and }|z|<1\} .
$$

Suppose also that $\mathscr{S} \subset \mathscr{A}$ consists of functions that are also univalent in $\Delta$.

For the facts that $0 \leqq \gamma<1$ and $f \in \mathscr{S}$ is as assumed in (1.1), we have the following subclasses of the normalized analytic function class $\mathscr{A}$ :

${ }^{*}$ Corresponding author.

E-mail addresses: harimsri@math.uvic.ca (H.M. Srivastava), gmsmoorthy@yahoo.com (G. Murugusundaramoorthy), shezaeldeeb@yahoo.com (S.M. El-Deeb).

Received November 14, 2020; Accepted December 16, 2020.

(C)2021 Journal of Nonlinear and Variational Analysis 
(1) $f$ is said to be in the class $\mathscr{S}^{*}(\gamma)$ of the starlike functions of order $\gamma$ in $\Delta$ (see [1]) if

$$
\mathscr{S}^{*}(\gamma):=\left\{f: f \in \mathscr{A} \quad \text { and } \quad \mathfrak{R}\left(\frac{z f^{\prime}(z)}{f(z)}\right)>\gamma \quad(z \in \Delta)\right\} .
$$

(2) $f$ is said to be in the class $\mathscr{K}(\gamma)$ of the convex functions of order $\gamma$ in $\Delta$ (see [1]) if

$$
\mathscr{K}(\gamma):=\left\{f: f \in \mathscr{A} \quad \text { and } \quad \Re\left(1+\frac{z f^{\prime \prime}(z)}{f^{\prime}(z)}\right)>\gamma \quad(z \in \Delta)\right\} .
$$

(3) $f$ is said to be in the class of the close-to-convex functions of order $\gamma$ in $\Delta$ (see [1] and [2]) if

$$
\mathscr{C}(\gamma):=\left\{f: f \in \mathscr{A} \quad \text { and } \quad \mathfrak{R}\left(\frac{z f^{\prime}(z)}{g(z)}\right)>\gamma \quad\left(g \in \mathscr{S}^{*}(0)=: \mathscr{S}^{*} ; z \in \Delta\right)\right\} .
$$

We note that

$$
\mathscr{S}^{*}(\gamma) \subset \mathscr{C}(\gamma) \subset \mathscr{S}
$$

and also that

$$
\left|a_{k}\right| \leqq k \quad(k \geqq 2)
$$

for $f \in \mathscr{S}$ by the Bieberbach conjecture (or de Brange's theorem) (see [1] and [3]).

The familiar Mittag-Leffler function $E_{\alpha}(z)$ and its two-parameter version $E_{\alpha, \beta}(z)$ are defined, respectively, by

$$
\begin{gathered}
E_{\alpha}(z):=\sum_{k=0}^{\infty} \frac{z^{k}}{\Gamma(\alpha k+1)} \quad \text { and } E_{\alpha, \beta}(z):=\sum_{k=0}^{\infty} \frac{z^{k}}{\Gamma(\alpha k+\beta)} \\
(z, \alpha, \beta \in \mathbb{C} ; \Re(\alpha)>0),
\end{gathered}
$$

which were first considered by Magnus Gustaf (Gösta) Mittag-Leffler (1846-1927) in 1903 and A. Wiman in 1905.

It is easily observed from (1.2) that

$$
E_{\alpha, \beta}(z)=\frac{1}{\Gamma(\beta)}+\sum_{k=2}^{\infty} \frac{z^{k-1}}{\Gamma(\alpha(k-1)+\beta)} \quad(z, \alpha, \beta \in \mathbb{C} ; \mathfrak{R}(\alpha)>0) .
$$

The Mittag-Leffler function $E_{\alpha}(z)$ and its two-parameter version $E_{\alpha, \beta}(z)$ are known to contain, as their special cases, a number of elementary functions such as the exponential, trigonometric and hyperbolic functions. In fact, these Mittag-Leffler functions happen to be the most commonly-used special cases of the Fox-Wright function ${ }_{p} \Phi_{q}(z)$ with $p$ numerator parameters and $q$ denominator parameters, which is defined by the following series (see, for example, [4, p. 67, Eq. (1.12.68)] and [5, p. 21, Eq. 1.2(38)]):

$$
{ }_{p} \Psi_{q}\left[\begin{array}{c}
\left(\alpha_{1}, A_{1}\right), \cdots,\left(\alpha_{p}, A_{p}\right) ; \\
\left(\beta_{1}, B_{1}\right), \cdots,\left(\beta_{q}, B_{q}\right) ;
\end{array}\right]:=\sum_{n=0}^{\infty} \frac{\prod_{j=1}^{p} \Gamma\left(\alpha_{j}+A_{j} n\right)}{\prod_{j=1}^{q} \Gamma\left(\beta_{j}+B_{j} n\right)} \frac{z^{n}}{n !} .
$$

Indeed, Srivastava [6] in his obituary of Charles Fox (1897-1977), Srivastava [6] remarked as follows (see, for details, [6, p. 68]):

Although a substantially more general integral function than that in (1.3) was studied earlier by George Neville Watson (1886-1965) in 1913, Fox's methods were an improvement over 
that of Bishop Ernest William Barnes (1874-1953) who, in 1907, had discussed the asymptotic expansions of the generalized hypergeometric function (1.3) in the familiar special case when

$$
A_{j}=1 \quad(j=1, \cdots, p) \quad \text { and } \quad B_{k}=1 \quad(k=1, \cdots, q)
$$

and differed from those of Watson mainly in that no appeal was made to the properties of certain inverse factorial series. It should be mentioned that Fox's methods were further generalized by Sir Edward Maitland Wright (1906-2005) in 1935 and 1940 in order to cover the case of the integral function in (1.3) when

$$
|\arg (-z)| \leqq \pi-\frac{1}{2} \pi \varepsilon \quad(0<\varepsilon \leqq 2) .
$$

By comparing the definitions in (1.2) and (1.3), it is easily seen that

$$
E_{\alpha, \beta}(z)={ }_{1} \Psi_{1}\left[\begin{array}{c}
(1,1) ; \\
z \\
(\beta, \alpha) ;
\end{array}\right]
$$

The Mittag-Leffler type functions arise naturally in the solution of various fractional-order differential, integral and integro-differential equations, in the investigations of the fractionalorder generalizations of kinetic equations, random walks and Lévy flights and super-diffusive transport, and in the study of complex systems. Several properties of the Mittag-Leffler type functions can be found in, for example, [4, 7, 8, 9, 10, 11, 12, 13, 14].

Since the Mittag-Leffler function $E_{\alpha, \beta}(z)$ does not belong to the normalized analytic function class $\mathscr{A}$, it is natural to consider the following normalized version of the Mittag-Leffler functions:

$$
\begin{gathered}
\mathfrak{E}_{\alpha, \beta}(z)=z \Gamma(\beta) E_{\alpha, \beta}(z)=z+\sum_{k=2}^{\infty} \frac{\Gamma(\beta)}{\Gamma(\alpha(k-1)+\beta)} z^{k} \\
\left(z, \alpha \in \mathbb{C} ; \mathfrak{R}(\alpha)>0 ; \beta \in \mathbb{C} \backslash \mathbb{Z}_{0}^{-}\right),
\end{gathered}
$$

where $\mathbb{Z}_{0}^{-}$denotes the set of non-positive integers. In this paper, we shall restrict our attention to the case that the parameters $\alpha$ and $\beta$ are real-valued and $z \in \Delta$.

We now recall that a discrete random variable $x$ is said to have a Borel distribution if it takes on the values $1,2,3, \cdots$ with the following probabilities:

$$
\frac{e^{-\lambda}}{1 !}, \frac{2 \lambda e^{-2 \lambda}}{2 !}, \frac{9 \lambda^{2} e^{-3 \lambda}}{3 !}, \cdots
$$

respectively, where $\lambda$ is the parameter involved. Recently, Wanas and Khuttar [15] introduced the Borel distribution (BD) whose probability mass function is given by

$$
\operatorname{Prob}\{x=\rho\}=\frac{(\rho \lambda)^{\rho-1} e^{-\lambda \rho}}{\rho !} \quad(\rho=1,2,3, \cdots) .
$$

Wanas and Khuttar [15] also introduced the following series $\mathscr{M}(\lambda ; z)$ whose coefficients are probabilities of the Borel distribution (BD):

$$
\mathscr{M}(\lambda ; z)=z+\sum_{k=2}^{\infty} \frac{[\lambda(k-1)]^{k-2} e^{-\lambda(k-1)}}{(k-1) !} z^{k} \quad(0<\lambda \leqq 1) .
$$


Murugusundaramoorthy and El-Deeb [16] studied the Mittag-Leffler type Borel distribution as follows:

$$
\mathscr{P}(\lambda, \alpha, \beta ; \rho)=\frac{(\lambda \rho)^{\rho-1}}{\Gamma(\alpha \rho+\beta) E_{\alpha, \beta}(\lambda \rho)} \quad(\rho=0,1,2, \cdots)
$$

where $E_{\alpha, \beta}(z)$ is the two-parameter Mittag-Leffler function defined in (1.2). Thus, by using (1.4) and (1.5), and by means of the Hadamard product (or convolution), we now define the Mittag-Leffler type Borel distribution series as follows:

$$
\mathscr{B}(\lambda, \alpha, \beta)(z)=z+\sum_{k=2}^{\infty} \frac{\Gamma(\lambda(k-1)+1)[\lambda(k-1)]^{k-2} e^{-\lambda(k-1)}}{(k-1) ! \mathfrak{E}_{\alpha, \beta}(\lambda(k-1)) \Gamma(\alpha(k-1)+\beta)} z^{k} \quad(0<\lambda \leqq 1) .
$$

Furthermore, by using the Hadamard product (or convolution), we define

$$
\begin{aligned}
\mathscr{B}(\lambda, \alpha, \beta) f(z) & =\mathscr{B}(\lambda, \alpha, \beta)(z) * f(z) \\
& =z+\sum_{k=2}^{\infty} \frac{\Gamma(\lambda(k-1)+1)[\lambda(k-1)]^{k-2} e^{-\lambda(k-1)}}{(k-1) ! \mathfrak{E}_{\alpha, \beta}(\lambda(k-1)) \Gamma(\alpha(k-1)+\beta)} a_{k} z^{k} \\
& =z+\sum_{k=2}^{\infty} \phi_{k} a_{k} z^{k} \quad\left(\alpha \in \mathbb{C} ; \mathfrak{R}(\alpha)>0 ; \beta \in \mathbb{C} \backslash \mathbb{Z}_{0}^{-} ; 0<\lambda \leqq 1\right),
\end{aligned}
$$

where the coefficients $\left\{\phi_{k}\right\}_{k=2}^{\infty}$ are given by

$$
\phi_{k}=\frac{\Gamma(\lambda(k-1)+1)[\lambda(k-1)]^{k-2} e^{-\lambda(k-1)}}{(k-1) ! \mathfrak{E}_{\alpha, \beta}(\lambda(k-1)) \Gamma(\alpha(k-1)+\beta)} .
$$

We now turn to the Koebe One-Quarter Theorem (see [1]) which states that the image of the open unit disk $\Delta$ under every univalent function $f \in \mathscr{S}$ contains the disk of radius $\frac{1}{4}$. Therefore, every function $f \in \mathscr{S}$ has an inverse $f^{-1}$ that satisfies the following relationship:

$$
f\left(f^{-1}(w)\right)=w \quad\left(|w|<r_{0}(f) ; r_{0}(f) \geqq \frac{1}{4}\right),
$$

where

$$
\begin{aligned}
g(w) & =f^{-1}(w)=w-a_{2} w^{2}+\left(2 a_{2}^{2}-a_{3}\right) w^{3}-\left(5 a_{2}^{3}-5 a_{2} a_{3}+a_{4}\right) w^{4}+\cdots \\
& =w+\sum_{m=2}^{\infty} A_{m} w^{m}
\end{aligned}
$$

By definition, a function $f \in \mathscr{A}$ is said to be bi-univalent in $\Delta$ if both $f$ and $f^{-1}$ are univalent in $\Delta$. Let $\Sigma$ denote the class of bi-univalent functions in $\Delta$ given by (1.1). The class of analytic and bi-univalent functions was first introduced by Lewin [17] who proved that $\left|a_{2}\right|<1.51$. Subsequently, Brannan and Clunie [18] improved Lewin's result to $\left|a_{2}\right|<\sqrt{2}$. In a latter investigation, Netanyahu [19] proved that $\left|a_{2}\right|<\frac{4}{3}$. It is known that the following functions:

$$
f_{1}(z)=\frac{z}{1-z}, \quad f_{2}(z)=\frac{1}{2} \log \left(\frac{1+z}{1-z}\right) \quad \text { and } \quad f_{3}(z)=-\log (1-z)
$$


with their corresponding inverses given by

$$
f_{1}^{-1}(w)=\frac{w}{1+w}, \quad f_{2}^{-1}(w)=\frac{e^{2 w}-1}{e^{2 w}+1} \quad \text { and } \quad f_{3}^{-1}(w)=\frac{e^{w}-1}{e^{w}}
$$

are the elements of the analytic and bi-univalent function class $\Sigma$ (see [20] and [21]). A brief history and interesting examples of functions in the analytic and bi-univalent function class $\Sigma$ can be found in (for example) [21] and [22]. In fact, this pioneering work by Srivastava et al. [21] actually revived and resuscitated the study of the analytic and bi-univalent function class $\Sigma$ in recent years.

Brannan and Taha [23] introduced certain subclasses of the bi-univalent function class $\Sigma$ similar to the familiar subclasses $\mathscr{S}^{*}(\gamma)$ and $\mathscr{K}(\gamma)$ of starlike and convex functions of order $\gamma$ $(0 \leqq \gamma<1)$ in $\Delta$, respectively (see [22]). Following Brannan and Taha [23], the classes $\mathscr{S}_{\Sigma}^{*}(\gamma)$ and $\mathscr{K}_{\Sigma}(\gamma)$ of bi-starlike functions of order $\gamma$ in $\Delta$ and bi-convex functions of order $\gamma$ in $\Delta$ $(0<\gamma \leqq 1)$, corresponding to the function classes $\mathscr{S}^{*}(\gamma)$ and $\mathscr{K}(\gamma)$, were also introduced analogously as defined below:

$$
\begin{array}{r}
\mathscr{S}_{\Sigma}^{*}(\gamma):=\left\{f: f \in \mathscr{A},\left|\arg \left(\frac{z f^{\prime}(z)}{f(z)}\right)\right|<\frac{\gamma \pi}{2} \quad(z \in \Delta)\right. \\
\left.\quad \text { and }\left|\arg \left(\frac{w \mathscr{F}^{\prime}(w)}{\mathscr{F}(w)}\right)\right|<\frac{\gamma \pi}{2} \quad(w \in \Delta)\right\},
\end{array}
$$

where the function $\mathscr{F}$ is the analytic extension of $f^{-1}$ to $\Delta$, given by

$$
\mathscr{F}(w)=w-a_{2} w^{2}+\left(2 a_{2}^{2}-a_{3}\right) w^{3}-\left(5 a_{2}^{3}-5 a_{2} a_{3}+a_{4}\right) w^{4}+\cdots \quad(w \in \Delta)
$$

and

$$
\begin{array}{r}
\mathscr{K}_{\Sigma}^{*}(\gamma):=\left\{f: f \in \mathscr{A},\left|\arg \left(1+\frac{z f^{\prime \prime}(z)}{f^{\prime}(z)}\right)\right|<\frac{\gamma \pi}{2} \quad(z \in \Delta)\right. \\
\left.\quad \text { and }\left|\arg \left(1+\frac{w \mathscr{F}^{\prime \prime}(w)}{\mathscr{F}^{\prime}(w)}\right)\right|<\frac{\gamma \pi}{2} \quad(w \in \Delta)\right\} .
\end{array}
$$

For each of the following bi-univalent function classes:

$$
\mathscr{S}_{\Sigma}^{*}(\gamma) \text { and } \mathscr{K}_{\Sigma}(\gamma)
$$

Brannon and Taha [23] found non-sharp estimates on the first two Taylor-Maclaurin coefficients $\left|a_{2}\right|$ and $\left|a_{3}\right|$ (see, for details, [23]; see also [21]) and [24]. This last rather pioneering work by Srivastava et al. [21] not only revived and resuscitated the study of the bi-univalent function class $\Sigma$ in recent years, but it also represents one of the most important studies of the bi-univalent functions and has inspired many investigations in this area including the present paper. Many other recent papers deal with the problems initiated in this pioneering work by Srivastava et al. [21] such as, for example, [25, 26, 27, 28, 29] (see also [30, 31, 32, 33] and the references to other related developments which are cited in each of these publications).

Motivated by the earlier work of Sakar and Güney [34], we define the following subclass of bi-close-to-convex functions $\mathscr{M}_{\Sigma}^{\alpha, \beta, \lambda}(\gamma, \vartheta)$ related with the Mittag-Leffler type Borel distribution. 
Definition 1.1. For $0 \leqq \vartheta \leqq 1, \alpha \in \mathbb{C}, \beta \in \mathbb{C} \backslash \mathbb{Z}_{0}^{-}, 0<\lambda \leqq 1$ and $0 \leqq \gamma \leqq 1$, a function $f$ is said to be in the class $\mathscr{M}_{\Sigma}^{\alpha, \beta, \lambda}(\gamma, \vartheta)$ if there exists a function $g$ is in $S^{*}$ such that

$$
\Re\left(\frac{z(\mathscr{B}(\lambda, \alpha, \beta) f(z))^{\prime}+\vartheta z^{2}(\mathscr{B}(\lambda, \alpha, \beta) f(z))^{\prime \prime}}{(1-\vartheta) \mathscr{B}(\lambda, \alpha, \beta) g(z)+\vartheta z(\mathscr{B}(\lambda, \alpha, \beta) g(z))^{\prime}}\right)>\gamma \quad(z \in \Delta)
$$

and

$$
\mathfrak{R}\left(\frac{w(\mathscr{B}(\lambda, \alpha, \beta) \mathscr{F}(w))^{\prime}+\vartheta w^{2}(\mathscr{B}(\lambda, \alpha, \beta) \mathscr{F}(w))^{\prime \prime}}{(1-\vartheta) \mathscr{B}(\lambda, \alpha, \beta) \mathscr{G}(w)+\vartheta w(\mathscr{B}(\lambda, \alpha, \beta) \mathscr{G}(w))^{\prime}}\right)>\gamma \quad(w \in \Delta),
$$

where the function $\mathscr{F}$ is the analytic extension of $f^{-1}$ to $\Delta$, which is given by (1.8), and $\mathscr{G}$ is the analytic extension of $g^{-1}$ to $\Delta$ as follows:

$$
\mathscr{G}(w)=w-b_{2} w^{2}+\left(2 b_{2}^{2}-b_{3}\right) w^{3}-\left(5 b_{2}^{3}-5 b_{2} b_{3}+b_{4}\right) w^{4}+\cdots \quad(w \in \Delta) .
$$

Example 1.1. For $\vartheta=0, \alpha \in \mathbb{C}, \beta \in \mathbb{C} \backslash \mathbb{Z}_{0}^{-}, 0<\lambda \leqq 1$ and $0 \leqq \gamma \leqq 1$, the function $f$ is in the class $\mathscr{C} \mathscr{S}_{\Sigma}^{\alpha, \beta, \lambda}(\gamma)$ if the function $g$ is in $\mathscr{S}^{*}$ such that

$$
\mathfrak{R}\left(\frac{z(\mathscr{B}(\lambda, \alpha, \beta) f(z))^{\prime}}{\mathscr{B}(\lambda, \alpha, \beta) g(z)}\right)>\gamma \quad(z \in \Delta)
$$

and

$$
\Re\left(\frac{w(\mathscr{B}(\lambda, \alpha, \beta) \mathscr{F}(w))^{\prime}}{\mathscr{B}(\lambda, \alpha, \beta) \mathscr{G}(w)}\right)>\gamma \quad(w \in \Delta),
$$

where the function $\mathscr{F}$ is given by (1.8) and the function $\mathscr{G}$ is given by (1.11).

Example 1.2. For $\vartheta=1, \alpha \in \mathbb{C}, \beta \in \mathbb{C} \backslash \mathbb{Z}_{0}^{-}, 0<\lambda \leqq 1$ and $0 \leqq \gamma \leqq 1$, the function $f$ is in the class $\mathscr{C} \mathscr{K}_{\Sigma}^{\alpha, \beta, \lambda}(\gamma)$ if the function $g$ is in $\mathscr{S}^{*}$ such that

$$
\mathfrak{R}\left(\frac{(\mathscr{B}(\lambda, \alpha, \beta) f(z))^{\prime}+z(\mathscr{B}(\lambda, \alpha, \beta) f(z))^{\prime \prime}}{(\mathscr{B}(\lambda, \alpha, \beta) g(z))^{\prime}}\right)>\gamma \quad(z \in \Delta)
$$

and

$$
\Re\left(\frac{(\mathscr{B}(\lambda, \alpha, \beta) \mathscr{F}(w))^{\prime}+w(\mathscr{B}(\lambda, \alpha, \beta) \mathscr{F}(w))^{\prime \prime}}{(\mathscr{B}(\lambda, \alpha, \beta) \mathscr{G}(w))^{\prime}}\right)>\gamma \quad(w \in \Delta),
$$

where the function $\mathscr{F}$ is given by (1.8) and the function $\mathscr{G}$ is given by (1.11).

By setting $b_{k}=a_{k}$, we can define a Pascu-type class of bi-univalent functions related with the Mittag-Leffler type Borel distribution as defined below.

Definition 1.2. For $0 \leqq \vartheta \leqq 1, \alpha \in \mathbb{C}, \beta \in \mathbb{C} \backslash \mathbb{Z}_{0}^{-}, 0<\lambda \leqq 1$ and $0 \leqq \gamma \leqq 1$, a function $f$ is said to be in the class $\mathscr{P}_{\Sigma}^{\alpha, \beta, \lambda}(\gamma, \vartheta)$ if

$$
\Re\left(\frac{z(\mathscr{B}(\lambda, \alpha, \beta) f(z))^{\prime}+\vartheta z^{2}(\mathscr{B}(\lambda, \alpha, \beta) f(z))^{\prime \prime}}{(1-\vartheta) \mathscr{B}(\lambda, \alpha, \beta) f(z)+\vartheta z(\mathscr{B}(\lambda, \alpha, \beta) f(z))^{\prime}}\right)>\gamma \quad(z \in \Delta)
$$

and

$$
\Re\left(\frac{w(\mathscr{B}(\lambda, \alpha, \beta) \mathscr{F}(w))^{\prime}+\vartheta w^{2}(\mathscr{B}(\lambda, \alpha, \beta) \mathscr{F}(w))^{\prime \prime}}{(1-\vartheta) \mathscr{B}(\lambda, \alpha, \beta) \mathscr{F}(w)+\vartheta w(\mathscr{B}(\lambda, \alpha, \beta) \mathscr{F}(w))^{\prime}}\right)>\gamma \quad(w \in \Delta) .
$$


Remark 1.1. Upon letting $\vartheta=0$ and $\vartheta=1$, we can deduce the new subclasses of $\mathscr{S}_{\Sigma}^{\alpha, \beta, \lambda}(\gamma)$ and $\mathscr{K}_{\Sigma}^{\alpha, \beta, \lambda}(\gamma)$, respectively.

Next, by using the Faber polynomial expansion of functions $f \in \mathscr{A}$ of the form (1.1), the coefficients of its inverse map can be expressed as follows (see [35]):

$$
\mathscr{F}(w)=f^{-1}(w)=w+\sum_{k=2}^{\infty} \frac{1}{k} \mathscr{K}_{k-1}^{-k}\left(a_{2}, a_{3}, \cdots\right) w^{k}=w+\sum_{k=2}^{\infty} A_{k} w^{k},
$$

where

$$
\begin{aligned}
\mathscr{K}_{k-1}^{-k}\left(a_{2}, a_{3}, \cdots\right)= & \frac{(-k) !}{(-2 k+1) !(k-1) !} a_{2}^{k-1}+\frac{(-k) !}{(2(-k+1)) !(k-3) !} a_{2}^{k-3} a_{3} \\
& +\frac{(-k) !}{(-2 k+3) !(k-4) !} a_{2}^{k-4} a_{4}+\frac{(-k) !}{(2(-k+2) !(k-5) !} a_{2}^{k-5}\left[a_{5}+(-k+2) a_{3}^{2}\right] \\
& +\frac{(-k) !}{(-2 k+5) !(k-6) !} a_{2}^{k-6}\left[a_{6}+(-2 k+5) a_{3} a_{4}\right]+\sum_{i \geqq 7} a_{2}^{k-i} U_{i},
\end{aligned}
$$

such that $U_{j}$ (with $7 \leqq j \leqq k$ ) is a homogeneous polynomial in the variables $a_{2}, a_{3}, \cdots, a_{k}$. In particular, the first three terms of $\mathscr{K}_{k-1}^{-k}$ are given by

$$
\begin{gathered}
\mathscr{K}_{1}^{-2}=-2 a_{2}, \\
\mathscr{K}_{2}^{-3}=3\left(2 a_{2}^{2}-a_{3}\right)
\end{gathered}
$$

and

$$
\mathscr{K}_{3}^{-4}=-4\left(5 a_{2}^{3}-5 a_{2} a_{3}+a_{4}\right) .
$$

Here, as usual, such expressions as (for example) $(-n)$ ! are to be interpreted symbolically by

$$
(-n) ! \equiv \Gamma(1-n):=(-n)(-n-1)(-n-2) \cdots \quad\left(n \in \mathbb{N}_{0}\right) .
$$

In general, an expansion of $\mathscr{K}_{m}^{-n}(n \in \mathbb{N})$ is recalled as follows (see [25, 36, 37, 38, 39, 40, 41]):

$$
\mathscr{K}_{k}^{-n}=n a_{k}+\frac{n(n-1)}{2} \mathscr{D}_{k}^{2}+\frac{n !}{3 !(n-3) !} \mathscr{D}_{k}^{3}+\cdots+\frac{n !}{k !(n-k) !} \mathscr{D}_{k}^{n},
$$

where

$$
\mathscr{D}_{k}^{n}=\mathscr{D}_{k}^{n}\left(a_{2}, a_{3}, \cdots\right)
$$

and

$$
\mathscr{D}_{k}^{p}\left(a_{1}, a_{2}, \cdots, a_{k}\right)=\sum_{k=1}^{\infty} \frac{p !}{i_{1} ! \cdots i_{k} !} a_{1}^{i_{1}} \cdots a_{k}^{i_{k}},
$$

in which $a_{1}=1$ and the sum is taken over all non-negative integers $i_{1}, \cdots, i_{k}$ satisfying the following condition:

$$
i_{1}+i_{2}+\cdots+i_{k}=p \quad \text { and } \quad i_{1}+2 i_{2}+\cdots+k i_{k}=k .
$$

Evidently, we have

$$
\mathscr{D}_{k}^{k}\left(a_{1}, a_{2}, \cdots, a_{k}\right)=a_{1}^{k} .
$$

The following lemma will be needed to prove our results. 
Carathéodory Lemma. [1] If $\phi \in \mathscr{P}$ is given by

$$
\phi(z)=1+\sum_{k=1}^{\infty} c_{k} z^{k} \quad(z \in \Delta)
$$

then $\left|c_{k}\right| \leqq 2$ for each $k \geqq 1$. This inequality is sharp for all integers $k \geqq 1$ when $\mathscr{P}$ is the family of all functions $\phi$, which are analytic in $\Delta$ and have positive real part in $\Delta$ with $\phi(0)=1$.

\section{TAYLOR-MACLAURIN COEFFICIENT Estimates}

We introduce a bound for the general coefficients of functions in $\mathscr{M}_{\Sigma}^{\alpha, \beta, \lambda}(\gamma, \vartheta)$.

Theorem 2.1. Let the function $f$ given by (1.1) belong to the following class:

$$
\begin{gathered}
\mathscr{M}_{\Sigma}^{\alpha, \beta, \lambda}(\gamma, \vartheta) \\
\left(0 \leqq \vartheta \leqq 1 ; \alpha \in \mathbb{C} ; \Re(\alpha)>0 ; \beta \in \mathbb{C} \backslash \mathbb{Z}_{0}^{-} ; 0<\lambda \leqq 1 ; 0 \leqq \gamma \leqq 1\right) .
\end{gathered}
$$

If $a_{m}=0$ for $2 \leqq m \leqq k-1$, then

$$
\left|a_{k}\right| \leqq \frac{2(1-\gamma)}{k[1+(k-1) \vartheta] \phi_{k}}+1
$$

where $\phi_{k}$ is defined by (1.7).

Proof. Suppose that $f \in \mathscr{M}_{\Sigma}^{\alpha, \beta, \lambda}(\gamma, \vartheta)$. Then there is a function $g$ given by

$$
g(z):=z+\sum_{k=2}^{\infty} b_{k} z^{k} \in \mathscr{S}^{*}
$$

such that

$$
\Re\left(\frac{z(\mathscr{B}(\lambda, \alpha, \beta) f(z))^{\prime}+\vartheta z^{2}(\mathscr{B}(\lambda, \alpha, \beta) f(z))^{\prime \prime}}{(1-\vartheta) \mathscr{B}(\lambda, \alpha, \beta) g(z)+\vartheta z(\mathscr{B}(\lambda, \alpha, \beta) g(z))^{\prime}}\right)>\gamma \quad(z \in \Delta) .
$$

Therefore, by using the above-mentioned Faber polynomial expansion, we have

$$
\begin{aligned}
& \frac{z(\mathscr{B}(\lambda, \alpha, \beta) f(z))^{\prime}+\vartheta z^{2}(\mathscr{B}(\lambda, \alpha, \beta) f(z))^{\prime \prime}}{(1-\vartheta) \mathscr{B}(\lambda, \alpha, \beta) g(z)+\vartheta z(\mathscr{B}(\lambda, \alpha, \beta) g(z))^{\prime}} \\
& \quad=1+\sum_{k=2}^{\infty}\left([1+\vartheta(k-1)] \phi_{k}\left(k a_{k}-b_{k}\right)+\sum_{t=1}^{k-2} \phi_{k}[1+(k-t-1) \vartheta] K_{t}^{-1}\right. \\
& \left.\quad \cdot\left[(1+\vartheta) b_{2},(1+2 \vartheta) b_{3}, \cdots,(1+t \vartheta) b_{t+1}\right] \cdot\left[(k-t) a_{k-t}-b_{k-t}\right]\right) z^{k-1} \quad(z \in \Delta) .
\end{aligned}
$$

Moreover, for the inverse map $\mathscr{F}=f^{-1}$, there exists a function $\mathscr{G}$ given by

$$
\mathscr{G}(w)=w+\sum_{k=2}^{\infty} B_{k} w^{k} \in \mathscr{S}^{*}
$$

such that

$$
\Re\left(\frac{w(\mathscr{B}(\lambda, \alpha, \beta) \mathscr{F}(w))^{\prime}+\vartheta w^{2}(\mathscr{B}(\lambda, \alpha, \beta) \mathscr{F}(w))^{\prime \prime}}{(1-\vartheta) \mathscr{B}(\lambda, \alpha, \beta) \mathscr{G}(w)+\vartheta w(\mathscr{B}(\lambda, \alpha, \beta) \mathscr{G}(w))^{\prime}}\right)>\gamma \quad(w \in \Delta) .
$$


The Faber polynomial series of the inverse map $\mathscr{F}=f^{-1}$ is given by

$$
\mathscr{F}(w)=w+\sum_{k=2}^{\infty} A_{k} w^{k}
$$

We thus find for $w \in \Delta$ that

$$
\begin{aligned}
& \frac{w(\mathscr{B}(\lambda, \alpha, \beta) \mathscr{F}(w))^{\prime}+\vartheta w^{2}(\mathscr{B}(\lambda, \alpha, \beta) \mathscr{F}(w))^{\prime \prime}}{(1-\vartheta) \mathscr{B}(\lambda, \alpha, \beta) \mathscr{G}(w)+\vartheta w(\mathscr{B}(\lambda, \alpha, \beta) \mathscr{G}(w))^{\prime}} \\
& \quad=1+\sum_{k=2}^{\infty}\left([1+\vartheta(k-1)] \phi_{k}\left(k A_{k}-B_{k}\right)+\sum_{t=1}^{k-2} \phi_{k}[1+(k-t-1) \vartheta] K_{t}^{-1}\right. \\
& \left.\quad \cdot\left[(1+\vartheta) B_{2},(1+2 \vartheta) B_{3}, \cdots,(1+t \vartheta) B_{t+1}\right] \cdot\left[(k-t) A_{k-t}-B_{k-t}\right]\right) w^{k-1}
\end{aligned}
$$

Since

$$
f \in \mathscr{M}_{\Sigma}^{\alpha, \beta, \lambda}(\gamma, \vartheta) \quad \text { and } \quad \mathscr{F}=f^{-1} \in \mathscr{M}_{\Sigma}^{\alpha, \beta, \lambda}(\gamma, \vartheta)
$$

we know that there are the following two functions with positive real parts:

$$
u(z)=1+\sum_{k=1}^{\infty} c_{k} z^{k} \quad \text { and } \quad v(w)=1+\sum_{k=1}^{\infty} d_{k} w^{k}
$$

where

$$
\Re(u(z))>0 \quad \text { and } \quad \mathfrak{R}(v(w))>0 \quad(z, w \in \Delta),
$$

such that

$$
\begin{aligned}
\frac{z}{(}(\mathscr{B}(\lambda, \alpha, \beta) f(z))^{\prime}+\vartheta z^{2}(\mathscr{B}(\lambda, \alpha, \beta) f(z))^{\prime \prime} \\
\quad=\gamma+(1-\gamma) u(z) \\
\quad=1+(1-\gamma) \sum_{k=1}^{\infty} c_{k} z^{k}
\end{aligned}
$$

and

$$
\begin{aligned}
& \frac{w(\mathscr{B}(\lambda, \alpha, \beta) \mathscr{F}(w))^{\prime}+\vartheta w^{2}(\mathscr{B}(\lambda, \alpha, \beta) \mathscr{F}(w))^{\prime \prime}}{(1-\vartheta) \mathscr{B}(\lambda, \alpha, \beta) \mathscr{G}(w)+\vartheta w(\mathscr{B}(\lambda, \alpha, \beta) \mathscr{G}(w))^{\prime}} \\
& \quad=\gamma+(1-\gamma) v(w) \\
& \quad=1+(1-\gamma) \sum_{k=1}^{\infty} d_{k} w^{k} .
\end{aligned}
$$

Now, under the assumption that $a_{m}=0$ for $0 \leqq m \leqq k-1$, we obtain $A_{k}=-a_{k}$. Therefore, by using (2.1) and comparing the corresponding coefficients in (2.3), we find that

$$
[1+\vartheta(k-1)] \phi_{k}\left(k a_{k}-b_{k}\right)=(1-\gamma) c_{k-1}
$$


Similarly, by using (2.2) in (2.4), we have

$$
\begin{aligned}
{[1} & +\vartheta(k-1)] \phi_{k}\left(k A_{k}-B_{k}\right) \\
& =(1-\gamma) d_{k-1}-[1+\vartheta(k-1)] \phi_{k}\left(k a_{k}-b_{k}\right) \\
& =(1-\gamma) c_{k-1}
\end{aligned}
$$

and

$$
-[1+\vartheta(k-1)] \phi_{k}\left(-k A_{k}-B_{k}\right)=(1-\gamma) d_{k-1} .
$$

Taking the moduli of each member of (2.7) and (2.8) for $\left|b_{k}\right| \leqq k$ and $\left|B_{k}\right| \leqq k$, and using the Carathéodory Lemma, we conclude that

$$
\left|a_{k}\right| \leqq \frac{2(1-\gamma)}{k[1+(k-1) \vartheta] \phi_{k}}+1
$$

where $\phi_{k}$ is defined by (1.7). This completes the proof of Theorem 2.11.

Corollary 2.1. Let the function $f$ given by (1.1) belong to the following class:

$$
\mathscr{C} \mathscr{S}_{\Sigma}^{\alpha, \beta, \lambda}(\gamma)
$$

$$
\left(\alpha \in \mathbb{C} ; \Re(\alpha)>0 ; \beta \in \mathbb{C} \backslash \mathbb{Z}_{0}^{-} ; 0<\lambda \leqq 1 ; 0 \leqq \gamma \leqq 1\right) .
$$

If $a_{m}=0$ for $2 \leqq m \leqq k-1$, then

$$
\left|a_{k}\right| \leqq \frac{2(1-\gamma)}{k \phi_{k}}+1
$$

where $\phi_{k}$ is defined by (1.7).

Corollary 2.2. Let the function $f$ given by (1.1) belong to the the following class:

$$
\begin{gathered}
\mathscr{C} \mathscr{K}_{\Sigma}^{\alpha, \beta, \lambda}(\gamma) \\
\left(\alpha \in \mathbb{C} ; \Re(\alpha)>0 ; \beta \in \mathbb{C} \backslash \mathbb{Z}_{0}^{-} ; 0<\lambda \leqq 1 ; 0 \leqq \gamma \leqq 1\right) .
\end{gathered}
$$

If $a_{m}=0$ for $2 \leqq m \leqq k-1$, then

$$
\left|a_{k}\right| \leqq \frac{2(1-\gamma)}{k^{2} \phi_{k}}+1
$$

where $\phi_{k}$ is defined by (1.7).

In particular, if $g(z)=f(z)$, we obtain the class $\mathscr{P}_{\Sigma}^{\alpha, \beta, \lambda}(\gamma, \vartheta)$, which is a subclass of the class $\mathscr{M}_{\Sigma}^{\alpha, \beta, \lambda}(\gamma, \vartheta)$. Our next theorem proves the Taylor-Maclaurin coefficient estimates of this subclass of bi-starlike functions.

Theorem 2.2. Let the function $f$ given by (1.1) belong the following class:

$$
\begin{gathered}
\mathscr{P}_{\Sigma}^{\alpha, \beta, \lambda}(\gamma, \vartheta) \\
\left(0 \leqq \vartheta \leqq 1 ; \alpha \in \mathbb{C} ; \mathfrak{R}(\alpha)>0 ; \beta \in \mathbb{C} \backslash \mathbb{Z}_{0}^{-} ; 0<\lambda \leqq 1 ; 0 \leqq \gamma \leqq 1\right) .
\end{gathered}
$$


Then

$$
\left|a_{2}\right| \leqq \begin{cases}\frac{2(1-\gamma)}{(1+\vartheta) \phi_{2}} & \left(0 \leqq \gamma<1-\frac{(1+\vartheta)^{2} \phi_{2}^{2}}{2\left(1+2 \vartheta-\vartheta^{2}\right) \phi_{3}}\right) \\ \sqrt{\frac{2(1-\gamma)}{\left(1+2 \vartheta-\vartheta^{2}\right) \phi_{3}}} & \left(1-\frac{(1+\vartheta)^{2} \phi_{2}^{2}}{2\left(1+2 \vartheta-\vartheta^{2}\right) \phi_{3}} \leqq \gamma<1\right)\end{cases}
$$

and

$$
\left|a_{3}\right| \leqq \begin{cases}\frac{2(1-\gamma)}{\left(1+2 \vartheta-\vartheta^{2}\right) \phi_{3}} & \left(0 \leqq \gamma<1-\frac{(1+\vartheta)^{2} \phi_{2}^{2}}{2\left(1+2 \vartheta-\vartheta^{2}\right) \phi_{3}}\right) \\ \left(\frac{1-\gamma}{1+2 \vartheta}\right) \cdot\left(\frac{1}{\phi_{3}}+\frac{2(1-\gamma)}{\phi_{2}^{2}}\right) & \left(1-\frac{(1+\vartheta)^{2} \phi_{2}^{2}}{2\left(1+2 \vartheta-\vartheta^{2}\right) \phi_{3}} \leqq \gamma<1\right)\end{cases}
$$

where $\phi_{k}$ is defined by (1.7).

Proof. Putting $n=2$ and $n=3$ in (2.5) and (2.6), we have

$$
\begin{gathered}
(1+\vartheta) \phi_{2} a_{2}=(1-\gamma) c_{1} \\
{\left[2(1+2 \vartheta) a_{3}-(1+\vartheta)^{2} a_{2}^{2}\right] \phi_{3}=(1-\gamma) c_{2}} \\
-(1+\vartheta) \phi_{2} a_{2}=(1-\gamma) d_{1}
\end{gathered}
$$

and

$$
\left[-2(1+2 \vartheta) a_{3}+\left(3+6 \vartheta-\vartheta^{2}\right) a_{2}^{2}\right] \phi_{3}=(1-\gamma) d_{2}
$$

From (2.11) and (2.13), by using the Carathéodory Lemma, we obtain

$$
\begin{aligned}
\left|a_{2}\right| & =\frac{(1-\gamma)\left|c_{1}\right|}{(1+\vartheta) \phi_{2}}=\frac{(1-\gamma)\left|d_{1}\right|}{(1+\vartheta) \phi_{2}} \\
& \leqq \frac{2(1-\gamma)}{(1+\vartheta) \phi_{2}}
\end{aligned}
$$

Also, from (2.12) and (2.14), we have

$$
2\left(1+2 \vartheta-\vartheta^{2}\right) \phi_{3} a_{2}^{2}=(1-\gamma)\left(c_{2}+d_{2}\right),
$$

so that

$$
a_{2}^{2}=\frac{(1-\gamma)\left(c_{2}+d_{2}\right)}{2\left(1+2 \vartheta-\vartheta^{2}\right) \phi_{3}}
$$

which, by virtue of the Carathéodory Lemma, yields

$$
\left|a_{2}\right| \leqq \sqrt{\frac{2(1-\gamma)}{\left(1+2 \vartheta-\vartheta^{2}\right) \phi_{3}}} .
$$

From (2.15) and (2.17), we obtain the desired estimate on the coefficient $\left|a_{3}\right|$, as asserted in (2.9). 
In order to find the bound on the coefficient $\left|a_{3}\right|$, we subtract (2.14) from (2.12). We then find that

$$
4(1+2 \vartheta) \phi_{3}\left(a_{3}-a_{2}^{2}\right)=(1-\gamma)\left(c_{2}-d_{2}\right)
$$

that is,

$$
a_{3}=a_{2}^{2}+\frac{(1-\gamma)\left(c_{2}-d_{2}\right)}{4(1+2 \vartheta) \phi_{3}} .
$$

Now, upon substituting the value of $a_{2}^{2}$ from (2.17) into (2.18) and using the Carathéodory Lemma, we obtain

$$
\left|a_{3}\right| \leqq \frac{2(1-\gamma)}{\left(1+2 \vartheta-\vartheta^{2}\right) \phi_{3}} .
$$

Moreover, if we substitute the value of $a_{2}^{2}$ from (2.11) into (2.12), we have

$$
a_{3}=\left(\frac{1-\gamma}{2(1+2 \vartheta)}\right) \cdot\left(\frac{c_{2}}{\phi_{3}}+\frac{(1-\gamma) c_{1}^{2}}{\phi_{2}^{2}}\right)
$$

Applying the Carathéodory Lemma once again, we get

$$
\left|a_{3}\right| \leqq\left(\frac{1-\gamma}{1+2 \vartheta}\right) \cdot\left(\frac{1}{\phi_{3}}+\frac{2(1-\gamma)}{\phi_{2}^{2}}\right) .
$$

Finally, by combining (2.19) and (2.20), we have the desired estimate on the coefficient $\left|a_{3}\right|$, as asserted in (2.10). Thus the proof of Theorem 2.2 is completed.

\section{FeKete-Szegö Type Inequalities}

Estimates and inequalities for the celebrated Fekete-Szegö functional have been investigated in the literature extensively recently; see, for example, [33, 42, 43, 44, 45] and the references therein. In this section, we derive Fekete-Szegö type inequalities for the bi-univalent function classes, which we introduced in Section 1. Our main result in this section (see Theorem 3.1 below) is the solution of the Fekete-Szegö problem for the class $\mathscr{P}_{\Sigma}^{\alpha, \beta, \lambda}(\gamma, \vartheta)$.

Theorem 3.1. Let the function $f$ given by (1.1) belong to the following class:

$$
\begin{gathered}
\mathscr{P}_{\Sigma}^{\alpha, \beta, \lambda}(\gamma, \vartheta) \\
\left(0 \leqq \vartheta \leqq 1 ; \alpha \in \mathbb{C} ; \mathfrak{R}(\alpha)>0 ; \beta \in \mathbb{C} \backslash \mathbb{Z}_{0}^{-} ; 0<\lambda \leqq 1 ; 0 \leqq \gamma \leqq 1\right) .
\end{gathered}
$$

Then

$$
\left|a_{3}-\mu a_{2}^{2}\right| \leqq \begin{cases}\frac{1-\gamma}{(1+2 \vartheta) \phi_{3}} & \left(0 \leqq|h(\mu)| \leqq \frac{1-\gamma}{4(1+2 \vartheta) \phi_{3}}\right) \\ 4|h(\mu)| & \left(|h(\mu)| \geqq \frac{1-\gamma}{4(1+2 \vartheta) \phi_{3}}\right)\end{cases}
$$

where

$$
h(\mu)=\frac{(1-\mu)(1-\gamma)}{2\left(1+2 \vartheta-\vartheta^{2}\right) \phi_{3}} \quad(\mu \in \mathbb{C}) .
$$


Proof. From (2.18) and (2.16), we obtain

$$
a_{3}-\mu a_{2}^{2}=(1-\mu) \frac{(1-\gamma)\left(c_{2}+d_{2}\right)}{2\left(1+2 \vartheta-\vartheta^{2}\right) \phi_{3}}+\frac{(1-\gamma)\left(c_{2}-d_{2}\right)}{4(1+2 \vartheta) \phi_{3}}
$$

so that

$$
\begin{aligned}
a_{3}-\mu a_{2}^{2}=( & \left.\frac{(1-\mu)(1-\gamma)}{2\left(1+2 \vartheta-\vartheta^{2}\right) \phi_{3}}+\frac{1-\gamma}{4(1+2 \vartheta) \phi_{3}}\right) c_{2} \\
& +\left(\frac{(1-\mu)(1-\gamma)}{2\left(1+2 \vartheta-\vartheta^{2}\right) \phi_{3}}-\frac{1-\gamma}{4(1+2 \vartheta) \phi_{3}}\right) d_{2}
\end{aligned}
$$

Therefore, we have

$$
a_{3}-\mu a_{2}^{2}=\left(h(\mu)+\frac{1-\gamma}{4(1+2 \vartheta) \phi_{3}}\right) c_{2}+\left(h(\mu)-\frac{1-\gamma}{4(1+2 \vartheta) \phi_{3}}\right) d_{2},
$$

where

$$
h(\mu)=\frac{(1-\mu)(1-\gamma)}{2\left(1+2 \vartheta-\vartheta^{2}\right) \phi_{3}} .
$$

Thus, by taking the modulus of each member of (3.3), we conclude that

$$
\left|a_{3}-\mu a_{2}^{2}\right| \leqq \begin{cases}\frac{1-\gamma}{(1+2 \vartheta) \phi_{3}} & \left(0 \leqq|h(\mu)| \leqq \frac{1-\gamma}{4(1+2 \vartheta) \phi_{3}}\right) \\ 4|h(\mu)| & \left(|h(\mu)| \geqq \frac{1-\gamma}{4(1+2 \vartheta) \phi_{3}}\right),\end{cases}
$$

which evidently completes the proof of Theorem 3.1.

By taking $\mu=1$ in Theorem 3.1, we deduce the following corollary.

Corollary 3.1. Let the function $f$ given by (1.1) belong to the following class:

$$
\begin{gathered}
\mathscr{P}_{\Sigma}^{\alpha, \beta, \lambda}(\gamma, \vartheta) \\
\left(0 \leqq \vartheta \leqq 1 ; \alpha \in \mathbb{C} ; \Re(\alpha)>0 ; \beta \in \mathbb{C} \backslash \mathbb{Z}_{0}^{-} ; 0<\lambda \leqq 1 ; 0 \leqq \gamma \leqq 1\right) .
\end{gathered}
$$

Then

$$
\left|a_{3}-a_{2}^{2}\right| \leqq \frac{1-\gamma}{(1+2 \vartheta) \phi_{3}}
$$

Remark 3.1. By setting $\vartheta=0$ and $\vartheta=1$ in Theorem 2.2 and in Theorem 3.1, one can easily get the initial coefficient estimates and the Fekete-Szegö type inequalities for the following bi-univalent function classes:

$$
\mathscr{C} \mathscr{S}_{\Sigma}^{\alpha, \beta, \lambda}(\gamma) \text { and } \mathscr{C} \mathscr{K}_{\Sigma}^{\alpha, \beta, \lambda}(\gamma)
$$

respectively. 


\section{CONCLUding REMARKS AND OBSERVATIONS}

In this paper, we made use of the Borel distribution series of the Mittag-Leffler type with a view and introduced a new class of the bi-close-to-convex functions defined in the open unit disk $\Delta$. We successively applied the Faber polynomial expansion method to determine the estimates for the general Taylor-Maclaurin coefficients of the functions belonging to this new class of bi-close-to-convex functions in $\Delta$. We derived the Fekete-Szegö type inequalities for the bi-close-to-convex function class which we have introduced here.

Recently, in his survey-cum-expository review article, Srivastava [46] demonstrated how the theories of the basic (or $q$-) calculus and the fractional $q$-calculus have significantly encouraged and motivated further developments in Geometric Function Theory of Complex Analysis, which also include such topics of analytic and bi-univalent functions as those involving (for example) $q$-starlike, $q$-convex, and other related $q$-function classes as well as the associated Fekete-Szegö type inequalities (see, for details, [38, 39, 47, 48, 49, 50]).

In conclusion, we choose to reiterate an important observation, which was presented in the recently-published review-cum-expository review article by Srivastava [46], who pointed out the fact that the results for the above-mentioned or new $q$-analogues can easily (and possibly trivially) be translated into the corresponding results for the so-called $(p, q)$-analogues (with $0<$ $|q|<p \leqq 1$ ) by applying some obvious parametric and argument variations with the additional parameter $p$ being redundant.

\section{REFERENCES}

[1] P. L. Duren, Univalent Functions, Grundlehren der Mathematischen Wissenschaften, Band 259, SpringerVerlag, New York, Berlin, Heidelberg and Tokyo, 1983.

[2] W. Kaplan, Close-to-convex schlicht functions, Michigan Math. J. 1 (1952), 169-185.

[3] L. de Branges, A proof of the Bieberbach conjecture, Acta Math. 154 (1985), 137-152.

[4] A. A. Kilbas, H. M. Srivastava, J. J. Trujillo, Theory and Applications of Fractional Differential Equations, North-Holland Mathematical Studies, Vol. 204, Elsevier (North-Holland) Science Publishers, Amsterdam, London and New York, 2006.

[5] H. M. Srivastava, P. W. Karlsson, Multiple Gaussian Hypergeometric Series, Halsted Press (Ellis Horwood Limited, Chichester), John Wiley and Sons, New York, Chichester, Brisbane and Toronto, 1985.

[6] H. M. Srivastava, Charles Fox, Bull. London Math. Soc. 12 (1980), 67-70.

[7] A. A. Attiya, Some applications of Mittag-Leffler function in the unit disk, Filomat, 30 (2016), 2075-2081.

[8] D. Bansal, J. K. Prajapat, Certain geometric properties of the Mittag-Leffler functions, Complex Var. Elliptic Equ. 61 (2016), 338-350.

[9] B. A. Frasin, An application of an operator associated with generalized Mittag-Leffler function, Konuralp J. Math. 7 (2019), 199-202.

[10] B. A. Frasin, T. Al-Hawary, F. Yousef, Some properties of a linear operator involving generalized MittagLeffler function, Stud. Univ. Babeş-Bolyai Math. 65 (2020), 67-75.

[11] R. Gorenflo, F. Mainardi, H. M. Srivastava, Special functions in fractional relaxation-oscillation and fractional diffusion-wave phenomena, In: Proceedings of the Eighth International Colloquium on Differential Equations (Plovdiv, Bulgaria; August 18-23, 1997) (D. Bainov, Editor), pp. 195-202, VSP Publishers, Utrecht and Tokyo, 1998.

[12] V. Kiryakova, Generalized Fractional Calculus and Applications, Pitman Research Notes in Mathematics, Vol. 301, Longman Scientific and Technical, Harlow (Essex), 1993.

[13] H. M. Srivastava, Fractional-order derivatives and integrals: Introductory overview and recent developments, Kyungpook Math. J. 60 (2020), 73-116.

[14] Ž. Tomovski, R. Hilfer, H. M. Srivastava, Fractional and operational calculus with generalized fractional derivative operators and Mittag-Leffler type functions, Integral Transforms Spec. Funct. 21 (2010), 797-814. 
[15] A. K. Wanas, J. A. Khuttar, Applications of Borel distribution series on analytic functions, Earthline J. Math. Sci. 4 (2020), 71-82.

[16] G. Murugusundaramoorthy, S. M. El-Deeb, Second Hankel determinant for a class of analytic functions of the Mittag-Leffler-type Borel distribution related with Legendre polynomials, Turkish World Math. Soc. J. Appl. Engrg. Math. (In Press).

[17] M. Lewin, On a coefficient problem for bi-univalent functions, Proc. Amer. Math. Soc. 18 (1967), 63-68.

[18] D. A. Brannan, J. G. Clunie (Editors), Aspects of Contemporary Complex Analysis, In: Proceedings of the NATO Advanced Study Institute (University of Durham, Durham, U.K.; July 1-20, 1979), pp. 79-95, Academic Press, New York and London, 1980.

[19] E. Netanyahu, The minimal distance of the image boundary from the origin and the second coefficient of a univalent function in $|z|<1$, Arch. Rational Mech. Anal. 32 (1969), 100-112.

[20] S. M. El-Deeb, T. Bulboacă, B. M. El-Matary, Maclaurin coefficient estimates of bi-univalent functions connected with the $q$-derivative, Mathematics, 8 (2020), Article ID 418.

[21] H. M. Srivastava, A. K. Mishra, P. Gochhayat, Certain subclasses of analytic and bi-univalent functions, Appl. Math. Lett. 23 (2010), 1188-1192.

[22] D. A. Brannan, J. Clunie, W. E. Kirwan, Coefficient estimates for a class of star-like functions, Canad. J. Math. 22 (1970), 476-485.

[23] D. A. Brannan, T. S. Taha, On some classes of bi-unvalent functions, In: Mathematical Analysis and Its Applications (Kuwait; February 18-21, 1985) (S. M. Mazhar, A. Hamoui and N. S. Faour, Editors), pp. 5360, KFAS Proceedings Series, Vol. 3, Pergamon Press (Elsevier Science Limited), Oxford, 1988; see also Studia Univ. Babeş-Bolyai Math. 31 (1986), 70-77.

[24] H. M. Srivastava, S. S. Eker, R. M. Ali, Coefficient bounds for a certain class of analytic and bi-univalent functions, Filomat, 29 (2015), 1839-1845.

[25] H. Aldweby, M. Darus, On a subclass of bi-univalent functions associated with the $q$-derivative operator, J. Math. Comput. Sci. 19 (1) (2019), 58-64.

[26] S. Elhaddad, M. Darus, Coefficient estimates for a subclass of bi-univalent functions defined by $q$-derivative operator, Mathematics, 8 (2020), Article ID 306.

[27] S. M. El-Deeb, Maclaurin coefficient estimates for new subclasses of bi-univalent functions connected with a $q$-analogue of Bessel function, Abstr. Appl. Anal. 2020 (2020), Article ID 8368951.

[28] H. Ö. Güney, G. Murugusundaramoorthy, H. M. Srivastava, The second Hankel determinant for a certain class of bi-close-to-convex functions, Results Math. 74 (2019), Article ID 93.

[29] P. N. Kamble, M. G. Shrigan, Coefficient estimates for a subclass of bi-univalent functions defined by Sălăgean type $q$-calculus operator, Kyungpook Math. J. 58 (2018), 677-688.

[30] Ş. Altinkaya, Bounds for a new subclass of bi-univalent functions subordinate to the Fibonacci numbers, Turkish J. Math. 44 (2020), 553-560.

[31] H. M. Srivastava, S. Gaboury, F. Ghanim, Coefficient estimates for a general subclass of analytic and biunivalent functions of the Ma-Minda type, Rev. Real Acad. Cienc. Exactas Fís. Natur. Ser. A Mat. (RACSAM), 112 (2018), 1157-1168.

[32] H. M. Srivastava, A. K. Wanas, Initial Maclaurin coefficient bounds for new subclasses of analytic and $m$-fold symmetric bi-univalent functions defined by a linear combination, Kyungpook Math. J. 59 (2019), 493-503.

[33] H. Tang, H. M. Srivastava, S. Sivasubramanian, P. Gurusamy, The Fekete-Szegö functional problems for some classes of $m$-fold symmetric bi-univalent functions, J. Math. Inequal. 10 (2016), 1063-1092.

[34] F. M. Sakar, H. Ö. Güeny, Coefficient bounds for a new subclass of analytic bi-close-to-convex functions by making use of Faber polynomial expansion, Turkish J. Math. 41 (2017), 888-895.

[35] G. Faber, Über polynomische Entwickelungen, Math. Ann. 57 (1903), 389-408.

[36] S. Bulut, Faber polynomial coefficient estimates for a subclass of analytic bi-univalent functions, Filomat, 30 (2016), 1567-1575.

[37] H. M. Srivastava, S. S. Eker, S. G. Hamidi, J. M. Jahangiri, Faber polynomial coefficient estimates for biunivalent functions defined by the Tremblay fractional derivative operator, Bull. Iran. Math. Soc. 44 (2018), 149-157. 
[38] H. M. Srivastava, S. M. El-Deeb, The Faber polynomial expansion method and the Taylor-Maclaurin coefficient estimates of bi-close-to-convex functions connected with the q-convolution, AIMS Math. 5 (2020), 7087-7106.

[39] H. M. Srivastava, S. Khan, Q. Z. Ahmad, N. Khan, S. Hussain, The Faber polynomial expansion method and its application to the general coefficient problem for some subclasses of bi-univalent functions associated with a certain $q$-integral operator, Stud. Univ. Babeş-Bolyai Math. 63 (2018), 419-436.

[40] H. M. Srivastava, A. Motamednezhad, E. A. Adegan, Faber polynomial coefficient estimates for bi-univalent functions defined by using differential subordination and a certain fractional derivative operator, Mathematics, 8 (2020), Article ID 172.

[41] H. M. Srivastava, F. M. Sakar, H. Ö. Güney, Some general coefficient estimates for a new class of analytic and bi-univalent functions defined by a linear combination, Filomat, 34 (2018), 1313-1322.

[42] B. Kowalczyk, A. Lecko, H. M. Srivastava, A note on the Fekete-Szegö problem for close-to-convex functions with respect to convex functions, Publ. Inst. Math. (Beograd) (Nouvelle Sér.) 101 (2017), 143-149.

[43] H. M. Srivastava, S. Hussain, A. Raziq, M. Raza, The Fekete-Szegö functional for a subclass of analytic functions associated with quasi-subordination, Carpathian J. Math. 34 (2018), 103-113.

[44] H. M. Srivastava, N. Khan, M. Darus, S. Khan, Q. Z. Ahmad, S. Hussain, Fekete-Szegö type problems and their applications for a subclass of $q$-starlike functions with respect to symmetrical points, Mathematics, 8 (2020), Article ID 842.

[45] H. M. Srivastava, A. K. Mishra, M. K. Das, The Fekete-Szegö problem for a subclass of close-to-convex functions, Complex Variables Theory Appl. 44 (2001), 145-163.

[46] H. M. Srivastava, Operators of basic (or $q$-) calculus and fractional $q$-calculus and their applications in geometric function theory of complex analysis, Iran. J. Sci. Technol. Trans. A: Sci. 44 (2020), 327-344.

[47] B. Khan, H. M. Srivastava, M. Tahir, M. Darus, Q. Z. Ahmad, N. Khan, Applications of a certain $q$-integral operator to the subclasses of analytic and bi-univalent functions, AIMS Math. 6 (2021), 1024-1039.

[48] M. S. U. Rehman, Q. Z. Ahmad, H. M. Srivastava, N. Khan, M. Darus, B. Khan, Applications of higher-order $q$-derivatives to the subclass of $q$-starlike functions associated with the Janowski functions, AIMS Math. 6 (2021), 1110-1125.

[49] H. M. Srivastava, Ş. Altinkaya, S. Yalçın, Hankel determinant for a subclass of bi-univalent functions defined by using a symmetric $q$-derivative operator, Filomat, 32 (2018), 503-516.

[50] H. M. Srivastava, N. Raza, E. S. A. AbuJarad, G. Srivastava, M. H. AbuJarad, Fekete-Szegö inequality for classes of $(p, q)$-starlike and $(p, q)$-convex functions, Rev. Real Acad. Cienc. Exactas Fís. Natur. Ser. A Mat. (RACSAM) 113 (2019), 3563-3584. 